\title{
Aortic root aneurysm with right atrial clot: a rare combination
}

\author{
Debmalya Saha $^{1}$, Kaushik Mukherjee ${ }^{1}$, and Amrita Guha ${ }^{1}$ \\ ${ }^{1}$ AMRI Hospital Dhakuria
}

December 27, 2021

\begin{abstract}
Though the incidence of aneurysms involving the aortic root and/or ascending aorta is common, the combination of aortic root aneurysm and the right atrial clot is extremely rare. No such case is reported in literature till date. This case report presents a 52-year gentleman who came to our emergency department with complaints of breathlessness, abdominal distention, pedal swelling, and decreased urine output with extremely poor general condition. After hemodynamic stabilization and preoperative optimization and workup, he was managed with Bentall procedure with right atrial clot removal. The immediate postoperative course was normal except for deranged liver function tests. The patient was discharged on postoperative day ten.
\end{abstract}

\section{INTRODUCTION}

Right atrial clot in the background of aortic root aneurysm is an uncommon occurrence. Here we will present a case of 52-year male with aortic root aneurysm and right atrial clot after informed-written consent and institutional ethical approval obtained.

\section{CASE REPORT}

A 52-year male with extremely poor general condition showed symptoms of breathlessness, abdominal distension, pedal swelling, and decreased urine output. On general examination, his heart rate was 140/minute, blood pressure of $95 / 30 \mathrm{~mm}$ of $\mathrm{Hg}$, respiratory rate of $35 / \mathrm{min}$, saturation of $90 \%$ at room air with presence of anasarca. During examination of cardiovascular system, a diastolic murmur was audible at aortic area. On examining the respiratory system, we got crepitations on both lung fields. A diagnosis of acute decompensated heart failure was made on clinical ground.

On routine blood investigations, total leucocyte count was high with polymorphic leukocytosis, and there was deranged LFTs and deranged kidney function tests.

On Chest X ray, we found cardiomegaly with prominent aortic knob along with increased para-hilar vascularity, bilateral blunted costophrenic angles. ECG showed low voltage waves with features of left ventricular diastolic overload. Bedside 2D trans-thoracic ECHO(TTE) revealed poor LVEF of $25 \%$, global hypokinesia, severe aortic regurgitation and dilatation of aortic root and ascending aorta (ascending aorta $6.2 \mathrm{~cm}$ ). CT angiography of heart and great vessels could not be done because of deranged renal function and respiratory distress of the patient.

The patient was managed conservatively initially with inotropes, judicious use of diuretics, IV antibiotics (renally safe), high flow oxygen and intermittent noninvasive positive pressure ventilation (NIPPV/BiPAP) and strict intake-output charting. 


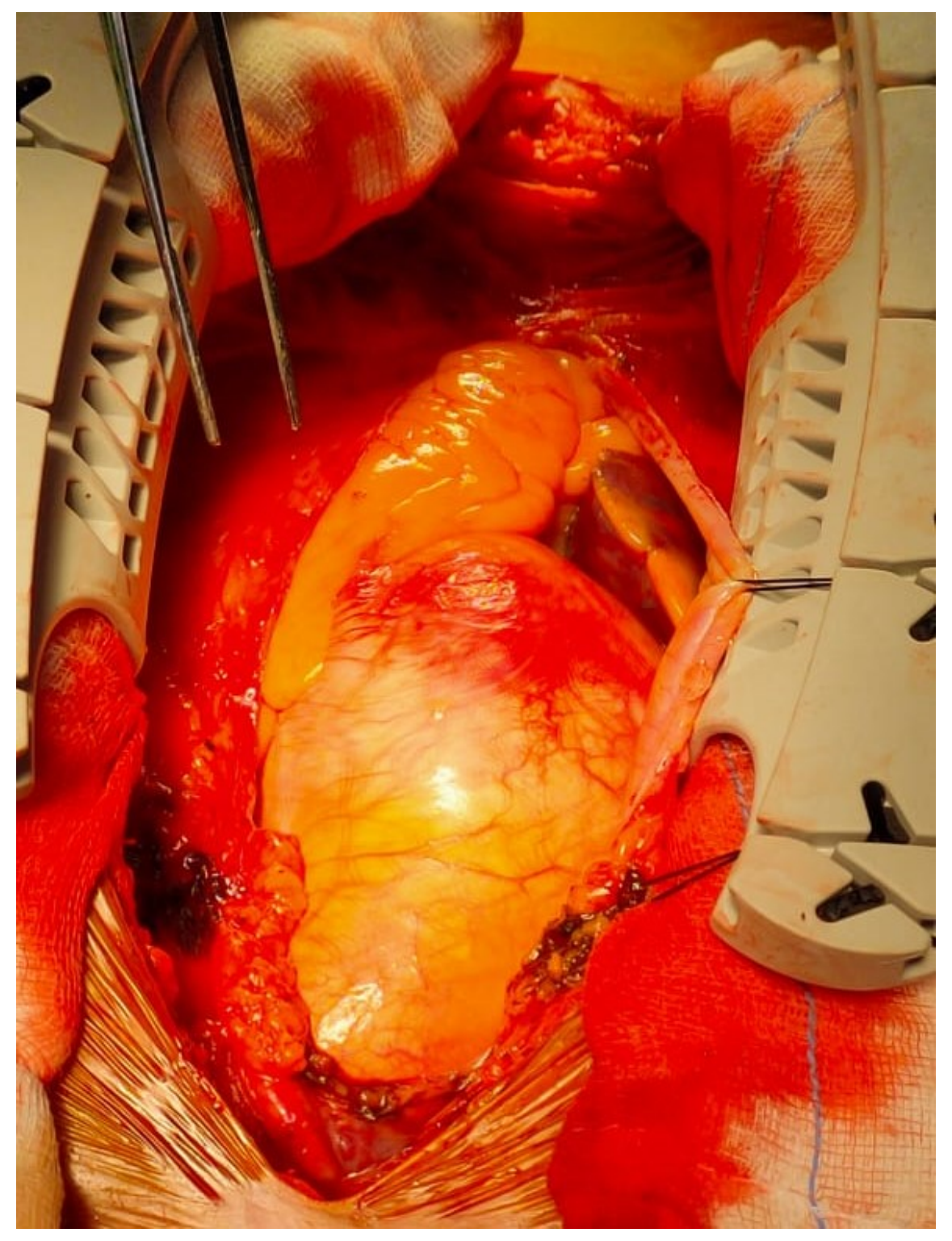




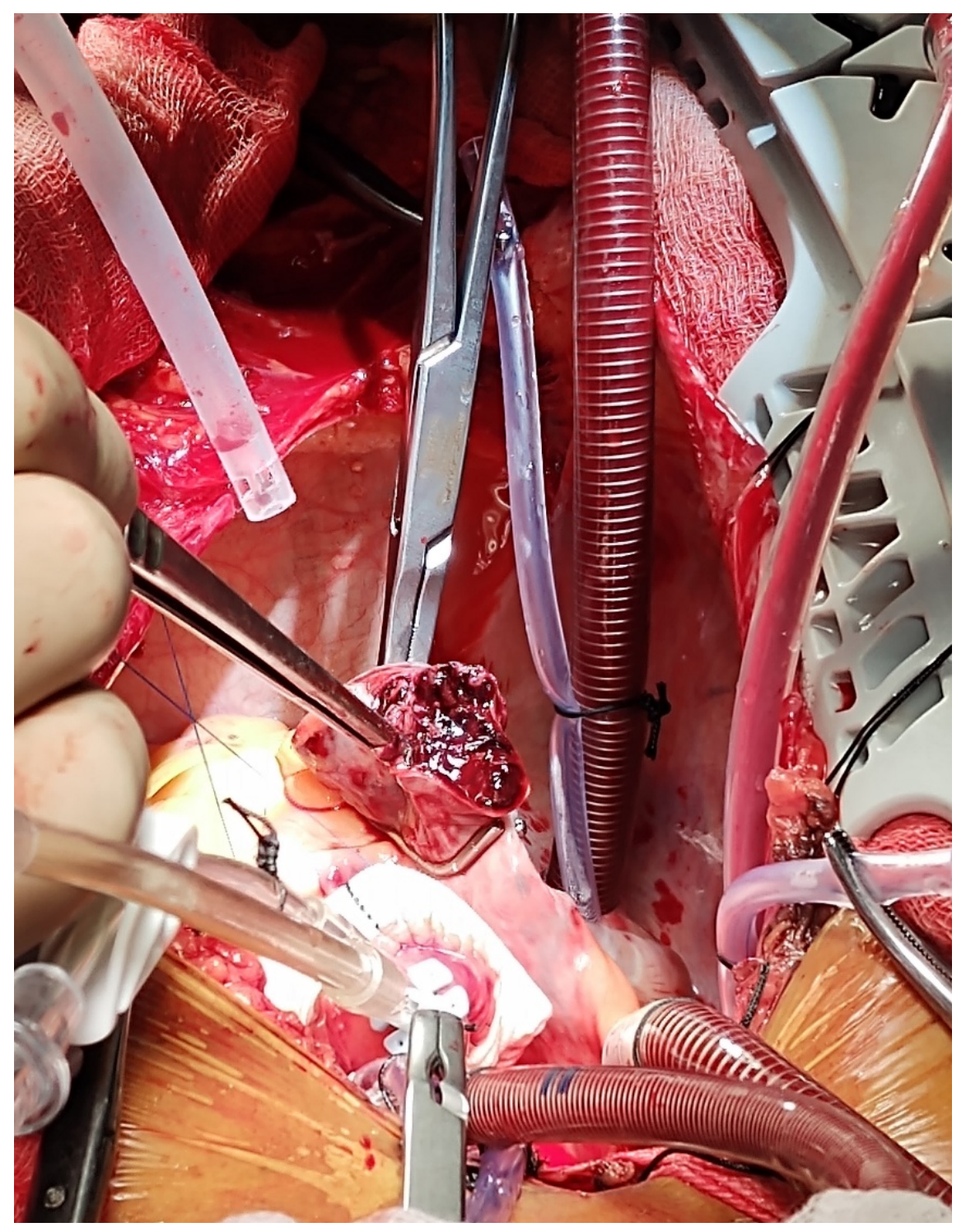

We parallelly planned for Bentall procedure on high-risk basis with ongoing resuscitation and stabilization. Our approach was via median sternotomy. The aortic root and ascending aorta were aneurysmal (Figure 1). Right atrial appendage was firm on palpation. Standard aorto-bicaval cannulation was done. Cardiopulmonary bypass was established. Aortic valve with ascending aorta was replaced with aortic valved conduit with re-implantation of coronary buttons. A large, organized clot occupying right atrial appendage (Figure 2) was removed.

Immediate post-operative period was uneventful other than deranged liver function tests which were in improving trend. The patient was discharged on post-operative day ten.

\section{CONCLUSION}

This type of rare association between ascending aortic aneurysm and right atrial clot must be reported in literature. This may alter the intra-operative strategy and subsequently can improve the operative outcome for this patient.

\section{CONFLICT OF INTERESTS}


The authors declare that there is no conflict of interest.

\section{ETHICS STATEMENT}

This study was approved by the Institutional Ethical Committee and the informed consent was taken from the patient's relatives in their own language.

\section{ORCID}

Debmalya Saha https://orcid.org/0000-0002-5350-4005

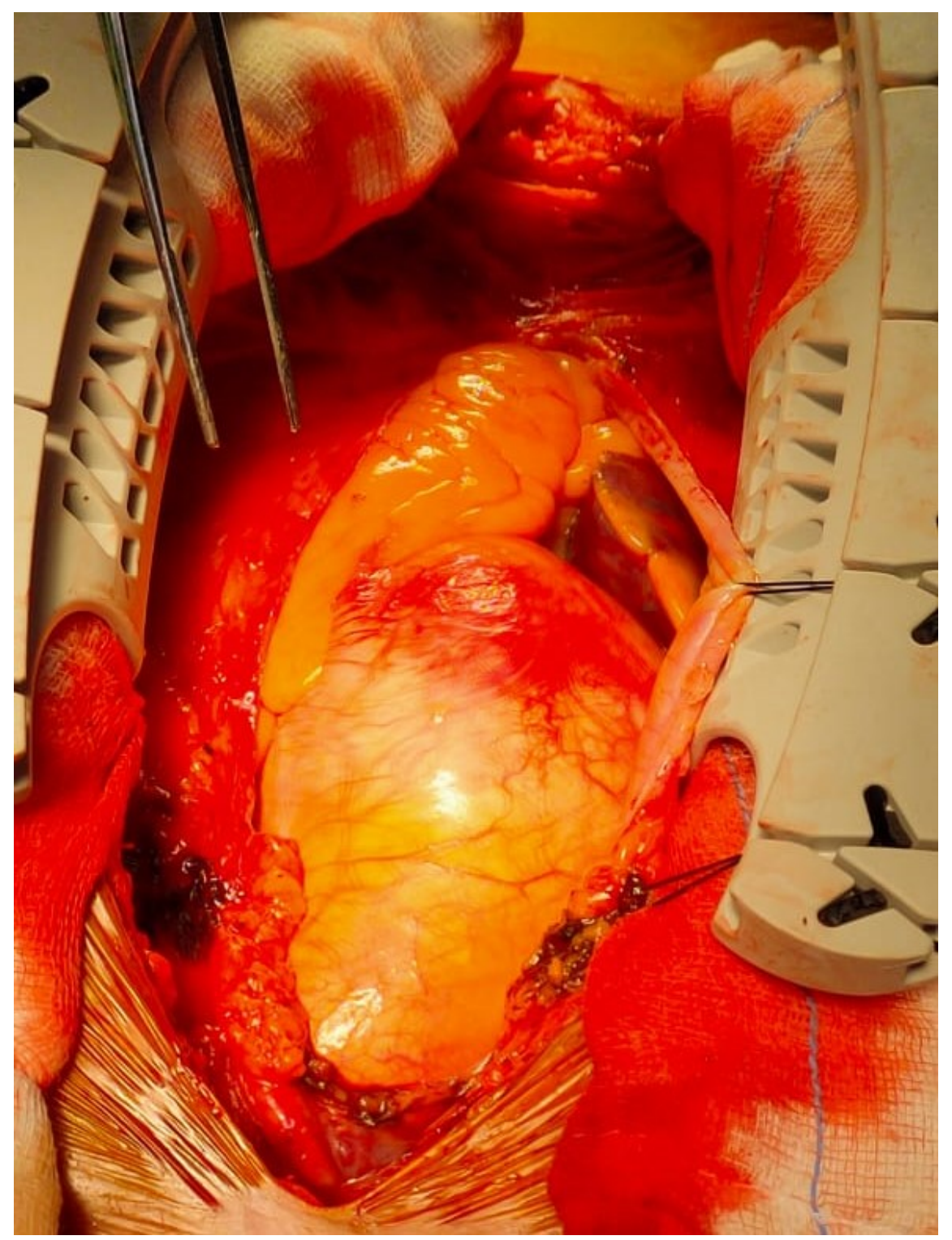




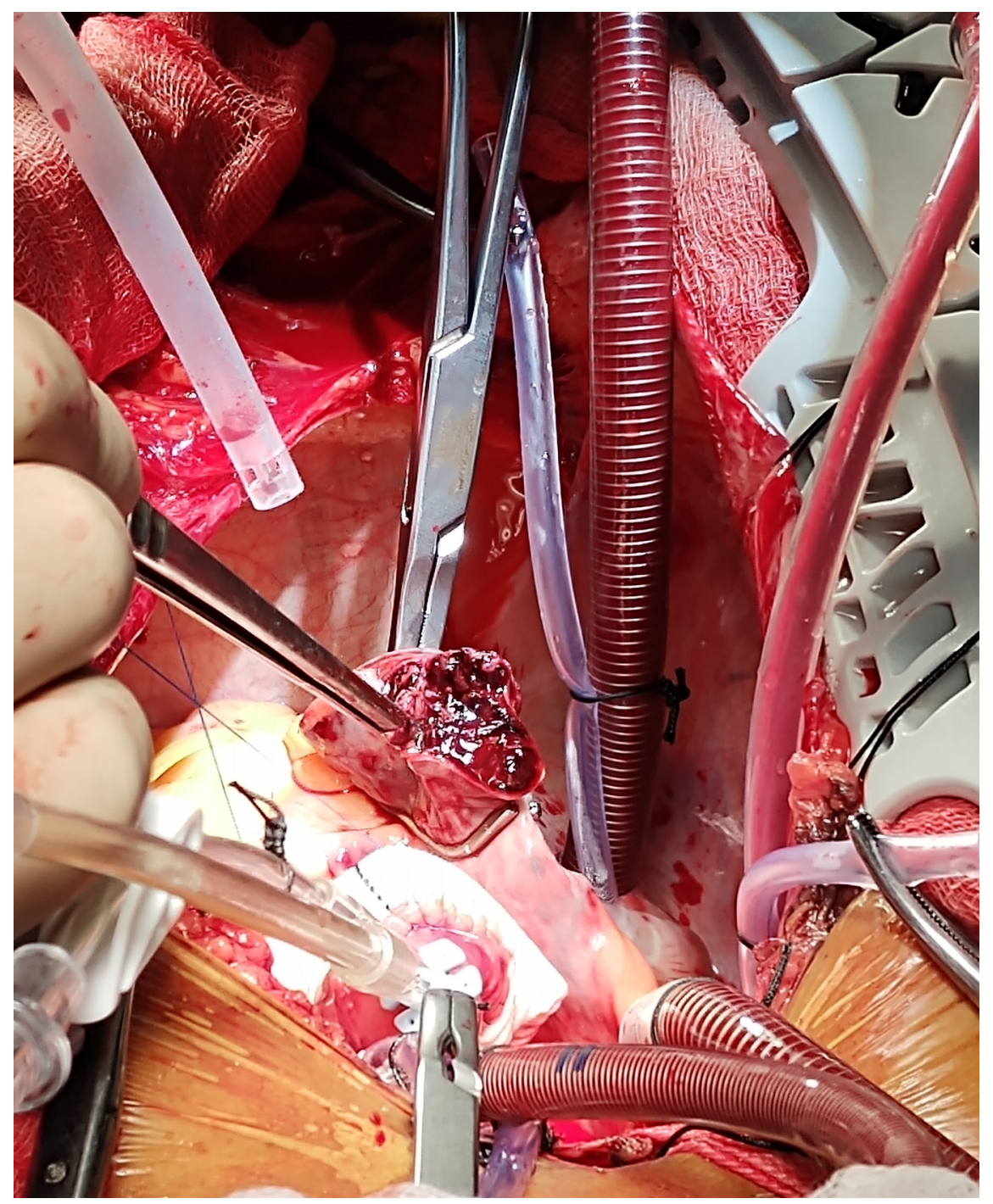

